\title{
Recent progress on few-body hypernuclei
}

\author{
Avraham $\mathrm{Gal}^{1, \star}$ \\ ${ }^{1}$ Racah Institute of Physics, the Hebrew University, Jerusalem 91904, Israel
}

\begin{abstract}
Few-body $\Lambda$ hypernuclei provide valuable information towards understanding strange matter. Recent experimental progress by the STAR Collaboration at the RHIC facility and by the ALICE Collaboration at the LHC has been matched by theoretical progress reviewed here: (i) lifetimes of the hypertriton ${ }_{\Lambda}^{3} \mathrm{H},{ }_{\Lambda}^{3} \mathrm{n}$ if particle-stable, ${ }_{\Lambda}^{4} \mathrm{H}$ and ${ }_{\Lambda}^{4} \mathrm{He}$ and their charge symmetry breaking, and (ii) the onset of $\Lambda \Lambda$ hypernuclear binding.
\end{abstract}

\section{Introduction}

Single- and double- $\Lambda$ hypernuclei provide unique extension of nuclear physics into strange hadronic matter [1]. Experimental data on $\Lambda$ and $\Lambda \Lambda$ hypernuclei are unfortunately poorer both in quantity and quality than the data available on normal nuclei. Nevertheless, the few dozen $\Lambda$ separation energies $B_{\Lambda}$ of single- $\Lambda$ hypernuclei $\left({ }_{\Lambda}^{\mathrm{A}} \mathrm{Z}\right)$ determined across the periodic table from $A=3$ to 208 , and the three $\Lambda \Lambda$ hypernuclei $\left({ }_{\Lambda \Lambda}^{\mathrm{A}} \mathrm{Z}\right)$ firmly established so far [2], provide useful testground for the role of strangeness in dense hadronic matter, say in neutron star matter. Particularly meaningful tests of hyperon-nucleon and hyperon-hyperon strong-interaction models are possible in light $\Lambda$ and $\Lambda \Lambda$ hypernuclei, $A \leq 6$, which the topics reviewed below are concerned with.

\section{$2{ }_{\Lambda}^{3} \mathrm{H}$ lifetime}

Measurements of the ${ }_{\Lambda}^{3} \mathrm{H}$ lifetime in emulsion or bubble-chamber experiments during the 1960s and early 1970s gave conflicting and puzzling results. Particularly troubling appeared a conference report by Block et al. claiming a lifetime of $\tau\left({ }_{\Lambda}^{3} \mathrm{H}\right)=\left(95_{-15}^{+19}\right)$ ps [3], to be compared with a free $\Lambda$ lifetime $\tau_{\Lambda}=(236 \pm 6)$ ps [4] measured in the same He bubble chamber (BC). However, the He BC experiment [5] concluding that era, coauthored by the same Block, reported a value of $\tau\left({ }_{\Lambda}^{3} \mathrm{H}\right)=\left(246_{-41}^{+62}\right) \mathrm{ps}$, in agreement with a $\Lambda$ lifetime of $(263 \pm 2)$ ps. Given a weakly bound $\Lambda, B_{\Lambda}\left({ }_{\Lambda}^{3} \mathrm{H}\right)=0.13 \pm 0.05 \mathrm{MeV}$ from emulsion studies [6], it was anticipated that $\tau\left({ }_{\Lambda}^{3} \mathrm{H}\right) \approx \tau_{\Lambda}$. Recent measurements of $\tau\left({ }_{\Lambda}^{3} \mathrm{H}\right)$ in relativistic heavy ion collision experiments renewed interest in the ${ }_{\Lambda}^{3} \mathrm{H}$ lifetime problem. The first round of results from STAR [7] and ALICE [8] suggested that $\tau\left({ }_{\Lambda}^{3} \mathrm{H}\right)$ is shorter than $\tau_{\Lambda}$ by as much as $(30 \pm 17) \%$. Whereas STAR's most recent published lifetime, from Au-Au collisions at $\sqrt{s_{\mathrm{NN}}}=7.7$ to $200 \mathrm{GeV}$, is even shorter than that: $\tau\left({ }_{\Lambda}^{3} \mathrm{H}\right)=142_{-21}^{+24} \pm 29 \mathrm{ps}$ [9], ALICE latest published lifetime, from $\mathrm{Pb}-\mathrm{Pb}$ collisions at $\sqrt{s_{\mathrm{NN}}}=5.02 \mathrm{TeV}$, is close to $\tau_{\Lambda}: \tau\left({ }_{\Lambda}^{3} \mathrm{H}\right)=242_{-38}^{+34} \pm 17$ ps [10], or $254 \pm 15 \pm 17 \mathrm{ps}$ as reported in ICHEP 2020 [11]. The latest news is that this STAR-ALICE apparent divergence has been

\footnotetext{
^avragal@savion.huji.ac.il, invited talk at the 19th International Conference on Strangeness in Quark Matter (SQM2021), Brookhaven National Laboratory, Upton, New York, USA (online), May 2021.
} 
resolved by new lifetime measurements in Au-Au collisions at $\sqrt{s_{\mathrm{NN}}}=3 \mathrm{GeV}$ reported by STAR at SQM2021: $\tau\left({ }_{\Lambda}^{3} \mathrm{H}\right)=231 \pm 29 \pm 37$ ps [12].

Table 1. Calculated hypertriton weak decay rates $\Gamma\left({ }_{\Lambda}^{3} \mathrm{H}_{\text {g.s. }}\right)$ in units of the free- $\Lambda$ decay rate $\Gamma_{\Lambda}$, using ${ }_{\Lambda}^{3} \mathrm{H}\left(\frac{1}{2}^{+}\right)$ wavefunctions that satisfy $B_{\Lambda}\left({ }_{\Lambda}^{3} \mathrm{H}\right)=0.13 \pm 0.05 \mathrm{MeV}$ from emulsion studies [6], and two-body branching ratios $\mathrm{R}_{3}=\Gamma\left({ }_{\Lambda}^{3} \mathrm{H} \rightarrow \pi^{-}+{ }^{3} \mathrm{He}\right) / \Gamma\left({ }_{\Lambda}^{3} \mathrm{H} \rightarrow \pi^{-}+\right.$all $)$. The underlined $\mathrm{R}_{3}$ value is the BC world-average experimental value [5].

\begin{tabular}{ccccc}
\hline Source & Method & $\pi \mathrm{FSI}$ & $\mathrm{R}_{3}$ & $\Gamma\left({ }_{\Lambda}^{3} \mathrm{H}\right) / \Gamma_{\Lambda}$ \\
\hline RD (1966) [13] & closure- $\Lambda$ pn & no & - & 1.14 \\
Congleton (1992) [14] & closure- $\Lambda \mathrm{d}$ & no & $0.33 \pm 0.02$ & 1.15 \\
Kamada (1998) [15] & Faddeev- $\Lambda$ pn & no & 0.379 & 1.06 \\
GG (2019) [16] & closure-Faddeev- $\Lambda$ pn & no & 0.362 & $1.11 \pm 0.01$ \\
HH (2020) [17] & $\pi$ EFT(LO)- $\Lambda$ d & no & $0.37 \pm 0.05$ & $0.98 \pm 0.15$ \\
\hline GG (2019) [16] & closure-Faddeev- $\Lambda$ pn & yes & 0.357 & $1.23 \pm 0.02$ \\
POGFG (2020) [18] & $\pi$ EFT(LO) $\Lambda$ pn $+\Sigma N N$ & yes & $\underline{0.35 \pm 0.04}$ & $1.38_{-0.14}^{+0.18}$ \\
\hline
\end{tabular}

A taste of what Theory has to say about the hypertriton lifetime is demonstrated by a representative selection of ${ }_{\Lambda}^{3} \mathrm{H}_{\text {g.s. }}\left(\frac{1}{2}^{+}\right)$lifetime calculations assembled in Table 1. Its upper part lists works that disregard pion final-state interaction $(\pi \mathrm{FSI})$. The closure approximation was used in Refs. $[13,14,16]$, whereas the other two works $[15,17]$ accounted microscopically for the outgoing nucleon phase space and FSI. The lifetimes $\tau\left({ }_{\Lambda}^{3} \mathrm{H}\right)=1 / \Gamma\left({ }_{\Lambda}^{3} \mathrm{H}\right)$ derived from this upper part are shorter than $\tau_{\Lambda}$ by less than $13 \%$. In contrast, the two works listed in the lower part of the table suggest ${ }_{\Lambda}^{3} \mathrm{H}$ lifetimes shorter than $\tau_{\Lambda}$ by more than $20 \%$, owing primarily to $\pi$ FSI where outgoing-pion plane waves are superseded by realistic pion distorted waves. This enhances $\Gamma\left({ }_{\Lambda}^{3} \mathrm{H}\right)$ by about $10 \%$ [16], or more realistically by $15 \%$ [18]. Interestingly, the recent $\pi$ EFT work [18] is the only one that bothered to address the small $\Sigma N N$ components, of order $\lesssim 1 \%$ probability, in the $\Lambda N N$ dominated ${ }_{\Lambda}^{3} \mathrm{H}$. A relatively large reduction of $\Gamma\left({ }_{\Lambda}^{3} \mathrm{H}\right)$ by $\approx 10 \%$ was found, owing to interference between the $\Sigma \rightarrow N \pi$ and $\Lambda \rightarrow N \pi$ weak-decay amplitudes. This effect was disregarded by Kamada et al. [15], thereby rendering their widely cited lifetime questionable.

The ${ }_{\Lambda}^{3} \mathrm{H}$ lifetime results shown in Table 1 hold generally for ${ }_{\Lambda}^{3} \mathrm{H}$ wavefunctions corresponding to given $B_{\Lambda}$ values within the emulsion $B_{\Lambda}\left({ }_{\Lambda}^{3} \mathrm{H}\right)=0.13 \pm 0.05 \mathrm{MeV}$ interval. The $B_{\Lambda}$ dependence of $\tau\left({ }_{\Lambda}^{3} \mathrm{H}\right)$ was studied in two of these works, with almost $B_{\Lambda}$-independent lifetime calculated in LO $\not$ EFT [17] and a rather strong $B_{\Lambda}$ dependence LO $\pi$ EFT [18], as shown in Fig. 1. The three red points in the figure correspond to three distinct values of $B_{\Lambda}\left({ }_{\Lambda}^{3} \mathrm{H}\right)$ reached by considering ultraviolet (UV) cutoff $\Lambda_{\mathrm{UV}}$ values smaller than $\Lambda_{\mathrm{UV}}=1200 \mathrm{MeV}$, beginning at which convergence is assured. This amounts to slightly varying the $\pi$ EFT short-range input. The resulting $B_{\Lambda}$ dependence of the computed twobody decay rate $\Gamma\left({ }_{\Lambda}^{3} \mathrm{H} \rightarrow{ }^{3} \mathrm{He}+\pi^{-}\right)$is satisfied by the blue points, all for $\Lambda_{\mathrm{UV}}=1200 \mathrm{MeV}$, obtained by varying systematically within allowed uncertainties some of the $\mathrm{NN}$ and $\mathrm{YN}$ chiral fit data [19]. Of the three red points, the middle one is that highlighted in Table 1 while the left one corresponds to $B_{\Lambda}\left({ }_{\Lambda}^{3} \mathrm{H}\right)=0.069 \mathrm{MeV}$ and $\tau\left({ }_{\Lambda}^{3} \mathrm{H}\right)=234 \pm 27 \mathrm{ps}$, a lifetime compatible with both ALICE and STAR new preliminary lifetime values $[11,12]$.

Given the strong correlation found in the $\pi$ EFT calculation [18] between $B_{\Lambda}\left({ }_{\Lambda}^{3} \mathrm{H}\right)$ and $\tau\left({ }_{\Lambda}^{3} \mathrm{H}\right), \mathrm{a}{ }_{\Lambda}^{3} \mathrm{H}$ lifetime as close to $\tau_{\Lambda}$ supports ALICE preliminary value $B_{\Lambda}\left({ }_{\Lambda}^{3} \mathrm{H}\right)=0.05 \pm 0.06 \pm 0.10 \mathrm{MeV}$ [20] over STAR's published value $0.41 \pm 0.12 \pm 0.11 \mathrm{MeV}$ [21]. As demonstrated in Ref. [18], going to as high values of $B_{\Lambda}\left({ }_{\Lambda}^{3} \mathrm{H}\right)$ would lead to considerably shorter values of $\tau\left({ }_{\Lambda}^{3} \mathrm{H}\right)$, which are compatible perhaps with STAR's published value [9], but are incompatible with STAR's new preliminary value [12]. 


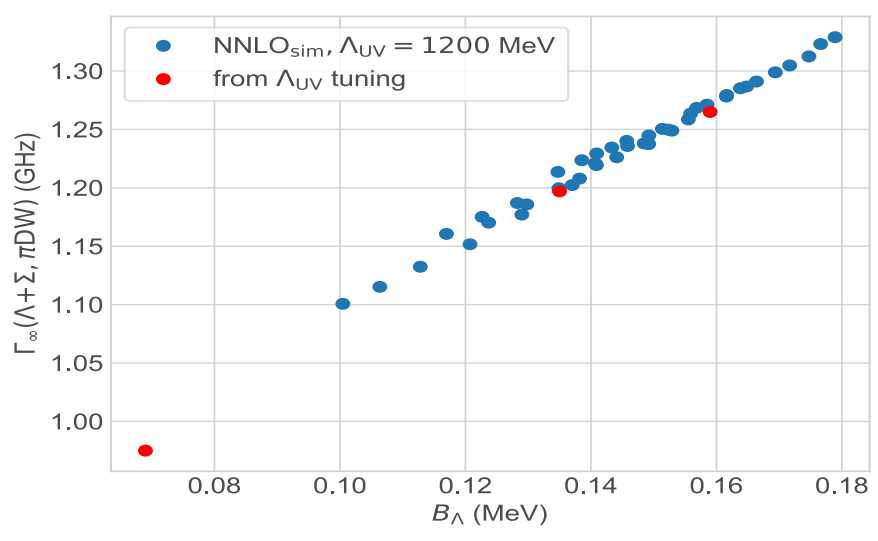

Figure 1. $\Gamma\left({ }_{\Lambda}^{3} \mathrm{H} \rightarrow \pi^{-}+{ }^{3} \mathrm{He}\right)$ vs. $B_{\Lambda}\left({ }_{\Lambda}^{3} \mathrm{H}\right)$ in $\pi$ EFT [19]. The three red points are from Ref. [18], see text.

\section{$3{ }_{\Lambda}^{3}$ n lifetime}

${ }_{\Lambda}^{3} \mathrm{n}$ was conjectured by the HypHI GSI Collaboration [22] to be particle stable, while unstable unanimously in recent theoretical calculations [23-25]. In ${ }_{\Lambda}^{3} \mathrm{n}$ decays induced by $\Lambda \rightarrow p+\pi^{-}$, where ${ }_{\Lambda}^{3} \mathrm{n}$ neutrons are spectators, the ${ }_{\Lambda}^{3} \mathrm{n} \rightarrow(p n n)+\pi^{-}$weak decay rate is given in the closure approximation essentially by the $\Lambda \rightarrow p+\pi^{-}$free-space weak-decay rate, whereas in $\Lambda \rightarrow n+\pi^{0}$ induced decays the production of a third low-momentum neutron is suppressed by the Pauli principle, so this ${ }_{\Lambda}^{3} \mathrm{n}$ weak decay branch may be disregarded up to perhaps a few percents. Hence $\Gamma\left({ }_{\Lambda}^{3} \mathrm{n}\right) / \Gamma_{\Lambda} \approx 1.114 \times 0.641=0.714$ [16], where the factor 1.114 follows from a difference between recoil energies in the ${ }_{\Lambda}^{3} \mathrm{n}$ and $\Lambda$ phase space factors, and the factor 0.641 is the free-space $\Lambda \rightarrow p+\pi^{-}$ fraction of the total $\Lambda \rightarrow N+\pi$ weak decay rate. This corresponds to a ${ }_{\Lambda}^{3} \mathrm{n}$ lifetime estimate of $\tau\left({ }_{\Lambda}^{3} \mathrm{n}\right) \approx 368 \mathrm{ps}$, considerably longer than $181_{-24}^{+30} \pm 25 \mathrm{ps}$ or $190_{-35}^{+47} \pm 36 \mathrm{ps}$ deduced from the $n d \pi^{-}$and $t \pi^{-}$alleged ${ }_{\Lambda}^{3} \mathrm{n}$ decay modes $[22,26]$, and thereby questioning the conjectured stability of ${ }_{\Lambda}^{3} \mathrm{n}$.

\section{$4{ }_{\Lambda}^{4} \mathrm{H}$ and ${ }_{\Lambda}^{4}$ He lifetimes}

A preliminary STAR lifetime value for ${ }_{\Lambda}^{4} \mathrm{H}$ was reported in this meeting: $\tau\left({ }_{\Lambda}^{4} \mathrm{H}\right)=218 \pm 8 \pm 12 \mathrm{ps}$ [12]. Do we understand this value in simple terms? A back-of-the-envelope estimate gives the following approximate expressions for ${ }_{\Lambda}^{4} \mathrm{H}(\mathrm{Z}=1)$ and ${ }_{\Lambda}^{4} \mathrm{He}(\mathrm{Z}=2)$ decay rates relative to the free $\Lambda$ decay:

$$
\Gamma\left({ }_{\Lambda}^{4} \mathrm{Z}\right) / \Gamma_{\Lambda} \approx(1+\eta(\bar{q})) \times\left(\alpha_{\mathrm{Z}} \times 0.7+1 \times 0.3\right)+0.20,
$$

where $\eta(\bar{q}) \approx 0.50 \pm 0.05$ is an exchange contribution to the dominant $s$-wave pionic weak decay rate, $\alpha_{\mathrm{Z}}=\frac{2}{3}\left(\frac{1}{3}\right)$ for $\mathrm{Z}=1(\mathrm{Z}=2)$ by applying the $\Delta I=\frac{1}{2}$ rule to the $\pi^{4}$ He two-body decay modes, $\mathrm{R}_{4}=0.7$ is the pionic two-body decay branching ratio [27], with $1-\mathrm{R}_{4}=0.3$ standing for the pionic multi-body part. Additional factors arising from recoil kinematics enhancement and $p$-wave decay suppression largely cancel out. Finally, the factor 0.20 stands for the observed $\Lambda N \rightarrow N N$ non-mesonic (n.m.) decay fraction $\Gamma_{\mathrm{n} . \mathrm{m} .} / \Gamma_{\Lambda}[28,29]$. The resulting $\tau\left({ }_{\Lambda}^{4} \mathrm{Z}\right)=1 / \Gamma\left({ }_{\Lambda}^{4} \mathrm{Z}\right)$ lifetime estimates

$$
\tau_{\text {th }}\left({ }_{\Lambda}^{4} \mathrm{H}\right)=195 \pm 10 \mathrm{ps}, \quad \tau_{\text {th }}\left({ }_{\Lambda}^{4} \mathrm{He}\right)=263 \pm 13 \mathrm{ps},
$$


with a 5\% assigned theoretical uncertainty, are in good agreement with $\tau_{\exp }\left({ }_{\Lambda}^{4} \mathrm{H}\right)=193 \pm 25$ ps and with $\tau_{\exp }\left({ }_{\Lambda}^{4} \mathrm{He}\right)=250 \pm 19$ ps as measured at KEK [28] and confirmed, for ${ }_{\Lambda}^{4} \mathrm{He}$, at BNL [29]. A preliminary value $\tau_{\exp }\left({ }_{\Lambda}^{4} \mathrm{H}\right)=180 \pm 7$ ps (stat. only) was derived in a test run of E73 at J-PARC [30].

\section{Charge symmetry breaking}

A special feature of the ${ }_{\Lambda}^{4} \mathrm{H}-{ }_{\Lambda}^{4} \mathrm{He}$ mirror hypernuclei is the particularly strong charge symmetry breaking (CSB) reflected in their spectra, as shown in Fig. 2. Although one-pion exchange (OPE) does not contribute directly to the $\Lambda N$ strong interaction owing to isospin invariance, it does contribute as pointed out by Dalitz and von Hippel (DvH) through a CSB potential $V_{\mathrm{CSB}}^{\mathrm{OPE}}$ generated by admixing the SU(3) octet $\Lambda_{I=0}$ and $\Sigma_{I=1}^{0}$ hyperons in the physical $\Lambda$ hyperon [34]. For the ${ }_{\Lambda}^{4} \mathrm{H}-{ }_{\Lambda}^{4} \mathrm{He}$ g.s. levels built on the mirror ${ }^{3} \mathrm{H}-{ }^{3} \mathrm{He}$ cores, and using $A=4$ wavefunctions generated within a LO $\pi$ EFT no-core shell-model calculation [35], the regularized Yukawa tail of $V_{\mathrm{CSB}}^{\mathrm{OPE}}$ gives rise to

$$
\mathrm{OPE}(\mathrm{DvH}): \Delta B_{\Lambda}^{J=0} \approx 175 \pm 40 \mathrm{keV}, \quad \Delta B_{\Lambda}^{J=1} \approx-50 \pm 10 \mathrm{keV},
$$

where $\Delta B_{\Lambda}^{J} \equiv B_{\Lambda}^{J}\left({ }_{\Lambda}^{4} \mathrm{He}\right)-B_{\Lambda}^{J}\left({ }_{\Lambda}^{4} \mathrm{H}\right)$. Remarkably, the large $\Delta B_{\Lambda}^{J=0}$ OPE CSB (central plus tensor) contribution to the splitting of the ${ }_{\Lambda}^{4} \mathrm{H}-{ }_{\Lambda}^{4} \mathrm{He}$ mirror g.s. levels roughly agrees with the observed value $\Delta B_{\Lambda}^{J=0}=233 \pm 92 \mathrm{keV}$ shown in Fig. 2 which is considerably larger than the $\approx 70 \mathrm{keV}$ CSB part of the Coulomb-dominated $\Delta B\left({ }^{3} \mathrm{H}-{ }^{3} \mathrm{He}\right)=764 \mathrm{keV}$ in the mirror core nuclei, driven apparently by shortrange $\rho^{0}-\omega$ mixing. Considerably smaller hypernuclear CSB contributions were found in $\Lambda N N N$ calculations by Coon et al. [36]: $\Delta B_{\Lambda}^{J=0}\left(\pi^{0} \eta+\rho^{0} \omega\right) \approx-20 \mathrm{keV}, \Delta B_{\Lambda}^{J=0}\left(\pi^{0} \eta+\rho^{0} \omega\right) \approx-10 \mathrm{keV}$.

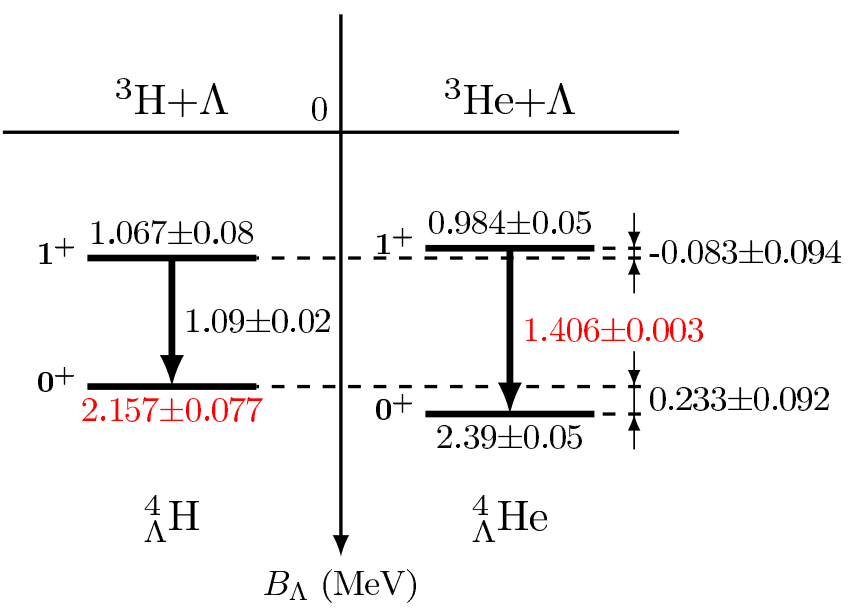

Figure 2. $A=4$ hypernuclear level scheme. Recent measurements of ${ }_{\Lambda}^{4} \mathrm{He}\left(1^{+}\right)$excitation energy [31] and of ${ }_{\Lambda}^{4} \mathrm{H}\left(0_{\text {g.s. }}^{+}\right)$binding energy [32] are marked in red. CSB splittings are shown to the right of the ${ }_{\Lambda}^{4} \mathrm{He}$ levels. Preliminary values from STAR are $\Delta B_{\Lambda}^{J=0}=0.13 \pm 0.13 \pm 0.07 \mathrm{MeV}, \Delta B_{\Lambda}^{J=1}=-0.19 \pm 0.13 \pm 0.07 \mathrm{MeV}$ [33].

\section{Onset of $\Lambda \Lambda$ hypernuclear binding}

Reliable data on $\Lambda \Lambda$ hypernuclei are scarce. the Nagara emulsion event [37, 38] identified unambiguously as ${ }_{\Lambda \Lambda}^{6} \mathrm{He}$, with $\Delta B_{\Lambda \Lambda}\left({ }_{\Lambda \Lambda}^{6} \mathrm{He}\right)=B_{\Lambda \Lambda}\left({ }_{\Lambda \Lambda}^{6} \mathrm{He}\right)-2 B_{\Lambda}\left({ }_{\Lambda}^{5} \mathrm{He}\right)=0.67 \pm 0.17 \mathrm{MeV}$ [38], is the lightest 
particle-stable $\Lambda \Lambda$ hypernucleus found so far. Are there lighter particle-stable $\Lambda \Lambda$ species? This question has been addressed recently in a LO $\not$ EFT calculation [39], sketched below, and in a full coupled-channel $\pi$ EFT calculation at NLO [40], both concluding that the onset of $\Lambda \Lambda$ hypernuclear binding is given by the isodoublet ${ }_{\Lambda \Lambda}^{5} \mathrm{H}-{ }_{\Lambda \Lambda}^{5} \mathrm{He}$.
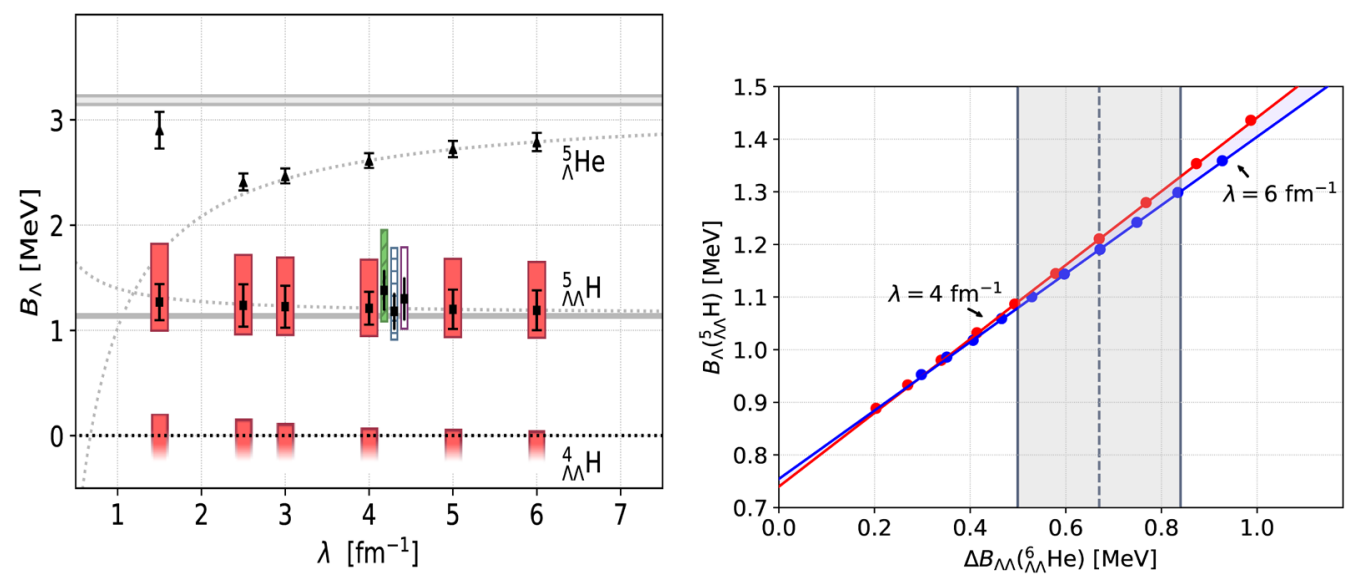

Figure 3. Left: $B_{\Lambda}\left({ }_{\Lambda}^{5} \mathrm{He},{ }_{\Lambda \Lambda}^{4} \mathrm{H},{ }_{\Lambda \Lambda}^{5} \mathrm{H}\right)$ from $\not$ EFT calculations [39]. Black error bars reflect given uncertainties in ${ }_{\Lambda}^{3} \mathrm{H},{ }_{\Lambda}^{4} \mathrm{H},{ }_{\Lambda}^{4} \mathrm{H}^{*},{ }_{\Lambda \Lambda}^{6} \mathrm{He} B_{\Lambda}$ input data, red rectangles arise by varying $a_{\Lambda \Lambda}$ between -0.5 to $-1.9 \mathrm{fm}$. Thin dotted lines show extrapolations to $\lambda \rightarrow \infty$ limits marked by gray horizontal bands. Right: Tjon lines calculations of $B_{\Lambda}\left({ }_{\Lambda \Lambda}^{5} \mathrm{H}\right)$ vs. $\Delta B_{\Lambda \Lambda}\left({ }_{\Lambda \Lambda}^{6} \mathrm{He}\right)$, with vertical straight lines marking the experimental uncertainty of $\Delta B_{\Lambda \Lambda}\left({ }_{\Lambda \Lambda}^{6} \mathrm{He}\right)$.

$\Lambda$ separation energy values $B_{\Lambda}\left({ }_{\Lambda \Lambda}^{5} \mathrm{H}\right)$ from $\not$ EFT calculations [39] are shown in the left panel of Fig. 3. Several representative values of the $\Lambda \Lambda$ scattering length were used from a broad range of values suggested by analyses of $\Lambda \Lambda$ correlations derived recently in relativistic heavyion collisions and by analyzing the KEK-PS E522 [41] invariant mass spectrum in the reaction ${ }^{12} \mathrm{C}\left(K^{-}, K^{+}\right) \Lambda \Lambda X$ near the $\Lambda \Lambda$ threshold; see Ref. [39] for references. Here the choice of $a_{\Lambda \Lambda}$ determines the one $\Lambda \Lambda$ low-energy constant (LEC) required at LO, while the $\Lambda \Lambda N$ LEC was fitted to the $\Delta B_{\Lambda \Lambda}\left({ }_{\Lambda \Lambda}^{6} \mathrm{He}\right)=0.67 \pm 0.17 \mathrm{MeV}$ datum [38]. The reported calculations used several $\Lambda N$ scatteringlength combinations, demonstrating that the $\Lambda N$ model dependence is weak when it comes to double$\Lambda$ hypernuclei, provided $B_{\Lambda}$ values of single- $\Lambda$ hypernuclei for $A<5$ are fitted to generate the necessary $\Lambda N N$ LECs. With values of $B_{\Lambda}\left({ }_{\Lambda \Lambda}^{5} \mathrm{H}\right) \sim 1 \mathrm{MeV}$, calculated over a broad range of cutoff values $\lambda$, it is clear that the particle stability of ${ }_{\Lambda \Lambda}^{5} \mathrm{H}$ is robust, in contrast to ${ }_{\Lambda \Lambda}^{4} \mathrm{H}$ for which $B_{\Lambda}\left({ }_{\Lambda \Lambda}^{4} \mathrm{H}\right)$ comes out negative over most of the permissible parameter space in these calculations. Finally, Tjon-line correlations [42] between $B_{\Lambda}\left({ }_{\Lambda \Lambda}^{5} \mathrm{H}\right)$ and $B_{\Lambda}\left({ }_{\Lambda \Lambda}^{6} \mathrm{He}\right)$ are demonstrated in the right panel of Fig. 3. We conclude that the isodoublet ${ }_{\Lambda \Lambda}^{5} \mathrm{H}-{ }_{\Lambda \Lambda}^{5} \mathrm{He}$ marks the onset of $\Lambda \Lambda$ hypernuclear binding. J-PARC Experiment E75 will search for ${ }_{\Lambda \Lambda}^{5} \mathrm{H}[43]$.

\section{Acknowledgments}

Discussions with my good colleagues coauthoring Refs. [16, 18, 35, 39] are gratefully acknowledged. This work is part of a project funded by the EU Horizon 2020 Research \& Innovation Programme under grant agreement 824093. 


\section{References}

[1] A. Gal, E.V. Hungerford, D.J. Millener, Rev. Mod. Phys. 88, 035004 (2016)

[2] A. Gal, D.J. Millener, Phys. Lett. B 701, 342 (2011)

[3] M.M. Block et al., Proc. Sienna Int'1. Conf. on Elementary Particles, Vol. 1, 63 (Bologna 1963)

[4] M.M. Block et al., Phys. Rev. 130, 766 (1963)

[5] G. Keyes, J. Sacton, J.H. Wickens, M.M. Block, Nucl. Phys. B 67, 269 (1973)

[6] D.H. Davis, Nucl. Phys. A 754, 3c (2005)

[7] B.I. Abelev et al. (STAR Collaboration), Science 328, 58 (2010)

[8] J. Adam et al. (ALICE Collaboration), Phys. Lett. B 754, 360 (2016)

[9] L. Adamczyk et al. (STAR Collaboration), Phys. Rev. C 97, 054909 (2018)

[10] S. Acharya et al. (ALICE Collaboration), Phys. Lett. B 797, 134905 (2019)

[11] F. Mazzaschi on behalf of the ALICE Collaboration, PoS 390 (ICHEP2020), 486 (2021)

[12] C. Hu on behalf of the STAR Collaboration, presented at SQM2021

[13] M. Rayet, R.H. Dalitz, Nuovo Cimento 46A, 786 (1966)

[14] G. Congleton, J. Phys. G 18, 339 (1992)

[15] H. Kamada, J. Golak, K. Miyagawa, H. Witala, W. Glöckle, Phys. Rev. C 57, 1595 (1998)

[16] A. Gal, H. Garcilazo, Phys. Lett. B 791, 48 (2019)

[17] F. Hildenbrand, H.-W. Hammer, Phys. Rev. C 102, 064002 (2020)

[18] A. Pérez-Obiol, D. Gazda, E. Friedman, A. Gal, Phys. Lett. B 811, 135916 (2020)

[19] A. Pérez-Obiol, D. Gazda, E. Friedman, A. Gal, in preparation (2021)

[20] P. Fecchio on behalf of the ALICE Collaboration, presented at SQM2021

[21] J. Adam et al. (STAR Collaboration), Nat. Phys. 16, 409 (2020)

[22] C. Rappold et al. (HypHI Collaboration), Phys. Rev. C 88, 041001(R) (2013)

[23] H. Garcilazo, A. Valcarce, Phys. Rev. C 89, 057001 (2014)

[24] E. Hiyama, S. Ohnishi, B.F. Gibson, Th.A. Rijken, Phys. Rev. C 89, 061302(R) (2014)

[25] A. Gal, H. Garcilazo, Phys. Lett. B 736, 93 (2014)

[26] T. Saito et al. (HypHI Collaboration), Nucl. Phys. A 954, 199 (2016)

[27] D. Bertrand et al., Nucl. Phys. B 16, 77 (1970)

[28] H. Outa et al., Nucl. Phys. A 639, 251c (1998)

[29] J.D. Parker et al., Phys. Rev. C 76, 035501 (2007)

[30] Y. Ma (J-PARC E73 status report), in https://kds.kek.jp/event/38719/

[31] T.O. Yamamoto et al. (J-PARC E13 Collaboration), Phys. Rev. Lett. 115, 222501 (2015)

[32] F. Schulz et al. (MAMI A1 Collaboration), Nucl. Phys. A 954, 149 (2016)

[33] T. Shao on behalf of the STAR Collaboration, presented at SQM2021

[34] R.H. Dalitz, F. von Hippel, Phys. Lett. 10, 153 (1964)

[35] D. Gazda, A. Gal, Phys. Rev. Lett. 116, 122501 (2016), Nucl. Phys. A 954, 161 (2016)

[36] S.A. Coon, H.K. Han, J. Carlson, B.F. Gibson, arXiv:nucl-th/9903034

[37] H. Takahashi et al., Phys. Rev. Lett. 87, 212502 (2001)

[38] J.K. Ahn et al. (KEK-PS E373 Collaboration), Phys. Rev. C 88, 014003 (2013)

[39] L. Contessi, M. Schäfer, N. Barnea, A. Gal, J. Mareš, Phys. Lett. B 797, 134893 (2019)

[40] H. Le, J. Haidenbauer, U.-G. Meißner, A. Nogga, Eur. Phys. J. A 57, 217 (2021)

[41] C.J. Yoon et al. (KEK-PS E522 Collaboration), Phys. Rev. C 75, 022201(R) (2007)

[42] J.A. Tjon, Phys. Lett. B 56, 217 (1975)

[43] H. Fujioka et al., Few-Body Syst. 62, 47 (2021) 